\title{
The exponential tracking and disturbance rejection of the propagated membrane action potential with conductance coefficient feedforward controllers
}

\author{
Weijiu Liu* \\ Department of Mathematics \\ University of Central Arkansas \\ 201 Donaghey Avenue, Conway, AR 72035, USA
}

\begin{abstract}
In the early 1950's, using their experimental data, Hodgkin and Huxley constructed the sodium and potassium conductance feedback controllers for their mathematical model of the flow of electric current through the surface membrane of a giant nerve fibre. In this paper, we re-formulate the construction as a problem of exponential tracking and disturbance rejection and then re-construct new conductance feedforward controllers in the more complicated case of a propagated action potential. The dynamics of the potential is governed by the Hodgkin-Huxley's partial differential equation (PDE) model. The problem is solved for any current disturbances and potential references and conductance coefficient feedforward controllers are designed by using the method of variable transform. It is proved that, under the designed feedforward controllers, the potential tracks exponentially a desired potential reference uniformly on an interval of one unit and the reference satisfies the controlled PDE model except an initial condition. A numerical example shows that the simulated action potential and sodium and potassium conductances are close to the experimental observations.
\end{abstract}

Keywords: Hodgkin-Huxley's mathematical model, coefficient feedforward controller, variable transform, exponential tracking, disturbance rejection, regulator equation.

\section{Introduction}

In the early 1950's, in a series of seminal papers $[11,12,13,14,15,16,17]$ concerned with the flow of electric current through the surface membrane of a giant nerve fibre, on the basis of their experimental data, Hodgkin and Huxley developed a famous mathematical model for the membrane potential of the giant nerve fibre:

$$
I=C_{m} \frac{d V}{d t}+G_{n a}\left(V-V_{n a}\right)+G_{k}\left(V-V_{k}\right)+\bar{g}_{l}\left(V-V_{l}\right),
$$

*Corresponding author. Email: weijiul@uca.edu, Phone: 1-501-450-5661, Fax: 1-501-450-5662 
where $I$ is the total membrane current, $C_{m}$ is the membrane capacity per unit area, $V$ is the displacement of the membrane potential from the absolute value of the resting potential, $V_{n a}, V_{k}$ and $V_{l}$ are the displacements of their equilibrium potentials from the resting potential for the sodium ions, potassium ions, and leakage ions made up by chloride and other ions, respectively, and $G_{n a}, G_{k}, \bar{g}_{l}$ are ionic conductances for the sodium ions, potassium ions, and leakage ions, respectively. The conductance $\bar{g}_{l}$ is assumed to be a positive constant. With their experimental data, they constructed the sodium and potassium conductances $G_{n a}, G_{k}$, which can be treated as dynamical feedback controllers, as follows (see, e.g., [14, 22]):

$$
\begin{aligned}
G_{n a} & =\bar{g}_{n a} h m^{3} \\
G_{k} & =\bar{g}_{k} n^{4} \\
\frac{d h}{d t} & =\alpha_{h}(1-h)-\beta_{h} h \\
\frac{d m}{d t} & =\alpha_{m}(1-m)-\beta_{m} m \\
\frac{d n}{d t} & =\alpha_{n}(1-n)-\beta_{n} n \\
\alpha_{h} & =0.07 \exp \left(\frac{-V}{20}\right) \\
\beta_{h} & =\frac{1}{\exp \left(\frac{30-V}{10}\right)+1} \\
\alpha_{m} & =\frac{0.1(25-V)}{\exp \left(\frac{25-V}{10}\right)-1} \\
\beta_{m} & =4 \exp \left(\frac{-V}{18}\right) \\
\alpha_{n} & =\frac{0.01(10-V)}{\exp \left(\frac{10-V}{10}\right)-1} \\
\beta_{n} & =0.125 \exp \left(\frac{-V}{80}\right)
\end{aligned}
$$

The units of potential, current density, conductance density, and capacitance density are $\mathrm{mV}, \mu \mathrm{A} / \mathrm{cm}^{2}$, $\mathrm{mS} / \mathrm{cm}^{2}, \mu \mathrm{F} / \mathrm{cm}^{2}$, respectively. In the case of membrane action potential in which the total membrane current $I$ is equal to zero, their numerical simulations showed that, under these feedback controllers, the simulated membrane potential $V$ agrees with the experimental data. However, these feedback controllers do not work when a small current disturbance may be present on the membrane.

The aim of this paper is to re-formulate the construction of the sodium and potassium conductances $G_{n a}, G_{k}$ as a problem of exponential tracking and disturbance rejection and then to re-construct these conductances in the more complicated case of a propagated action potential. In this case, the total 
membrane current $I$ is no longer equal to zero and is given by (see [14])

$$
I=\frac{a}{2 R} \frac{\partial^{2} V}{\partial x^{2}}
$$

where $a$ is the radius of the fibre and $R$ is the specific resistance of the axoplasm. In addition, the membrane may be subject to a small current disturbance $I_{d}(x, t)$. Therefore, the Hodgkin-Huxley's model for the membrane potential over one unit length of the fibre is given by

$$
\begin{aligned}
C_{m} \frac{\partial V}{\partial t} & =\frac{a}{2 R} \frac{\partial^{2} V}{\partial x^{2}}-G_{n a}\left(V-V_{n a}\right)-G_{k}\left(V-V_{k}\right)-\bar{g}_{l}\left(V-V_{l}\right)+I_{d}, \\
\frac{\partial V}{\partial x}(0, t) & =r_{1}, \quad \frac{\partial V}{\partial x}(1, t)=r_{2},
\end{aligned}
$$

where $r_{1}$ and $r_{2}$ are constants. In addition to the Neumann boundary condition on $V$, we will also consider the Dirichlet boundary condition:

$$
V(0, t)=V_{1}, \quad V(1, t)=V_{2}
$$

where $V_{1}$ and $V_{2}$ are constants. Let $V_{r}(x, t)$ be a biologically intrinsic membrane action potential reference pattern. To maintain the normal functioning of the giant nerve fibre, it could be essential for the potential $V(x, t)$ to track $V_{r}(x, t)$. Thus we introduce the error

$$
e(x, t)=V(x, t)-V_{r}(x, t) .
$$

Then the problem of exponential tracking and disturbance rejection of the membrane potential is to design coefficient feedforward controllers $G_{n a}$ and $G_{k}$ such that

$$
\max _{0 \leq x \leq 1}|e(x, t)| \leq C e^{-\lambda t}
$$

where $C$ and $\lambda$ are positive constants.

As a famous and important mathematical model, it is well known that the Hodgkin-Huxley's model (14) has been extensively studied (see, e.g., $[2,5,9,10,20,35]$ ). As a parabolic partial differential equation, the problem of asymptotic tracking and disturbance rejection with a boundary controller or a controller in the equation as an independent term has been also extensively investigated (see, e.g., $[4,6,7,28,29,30,31,32,33,34])$. However, to my knowledge, the problem of exponential tracking with the coefficient feedforward controllers has not been studied yet.

Using the method of signal dependent variable transform used in $[3,18,21,23,24,25,26]$, we transform the tracking problem into two separate problems: a regulator equation and a partial differential equation with the zero equilibrium. The regulator equation can be solved explicitly, its solution is substituted into the conductance coefficients of the partial differential equation, and then feedforward controllers are derived 
by making the conductance coefficients negative so that the partial differential equation is exponentially stable. It is proved that, under the designed feedforward controllers, the potential tracks exponentially a desired potential reference uniformly on an interval of one unit and the reference satisfies the controlled PDE model except an initial condition. A numerical example shows that the simulated action potential and sodium and potassium conductances are close to the experimental observations.

\section{Exponential tracking}

In what follows, $H^{s}(0,1)$ denotes the usual Sobolev space (see [1]) for any $s \in \mathbb{R}$. For $s \geq 0, H_{0}^{s}(0,1)$ denotes the completion of $C_{0}^{\infty}(0,1)$ in $H^{s}(0,1)$, where $C_{0}^{\infty}(0,1)$ denotes the space of all infinitely differentiable functions on $(0,1)$ with compact support in $(0,1)$. $C[0,1]$ denotes the space of all continuous functions on $[0,1]$ with the maximum norm. The norms of $H^{s}(0,1)$ and $C[0,1]$ are denoted by $\|\cdot\|_{H^{s}}$ and $\|\cdot\|_{C}$, respectively.

For the convenience, we combine the equation (14) with the error equation (16) as follows:

$$
\begin{aligned}
C_{m} \frac{\partial V}{\partial t} & =\frac{a}{2 R} \frac{\partial^{2} V}{\partial x^{2}}-G_{n a}\left(V-V_{n a}\right)-G_{k}\left(V-V_{k}\right)-\bar{g}_{l}\left(V-V_{l}\right)+I_{d}, \\
\frac{\partial V}{\partial x}(0, t) & =r_{1}, \quad \frac{\partial V}{\partial x}(1, t)=r_{2}, \\
e(x, t) & =V(x, t)-V_{r}(x, t), \\
V(x, 0) & =V_{0}(x) .
\end{aligned}
$$

Like the finite dimensional control systems [27], this problem of exponential tracking and disturbance rejection can be solved for any disturbances and references and they are not required to be governed by an exogenous system.

Theorem 2.1. Assume that $\lambda>0$ and the initial condition $V_{0} \in H^{1}(0,1)$. Suppose that

1. The disturbance function $I_{d}(x, t)$ is continuous.

2. The potential reference function $V_{r}(x, t)$ is continuously differentiable in $t$ and twice continuously differentiable in $x$ and satisfies the boundary condition

$$
\frac{\partial V_{r}}{\partial x}(0, t)=r_{1}, \quad \frac{\partial V_{r}}{\partial x}(1, t)=r_{2}
$$

Then, under the feedforward controllers:

$$
\begin{aligned}
G_{k} & =\left[\left(\lambda-\bar{g}_{l}\right)\left(V_{r}-V_{n a}\right)-\frac{a}{2 R} \frac{\partial^{2} V_{r}}{\partial x^{2}}-I_{d}+C_{m} \frac{\partial V_{r}}{\partial t}+\bar{g}_{l}\left(V_{r}-V_{l}\right)\right] /\left(V_{k}-V_{n a}\right) \\
G_{n a} & =\left[\frac{a}{2 R} \frac{\partial^{2} V_{r}}{\partial x^{2}}+I_{d}-C_{m} \frac{\partial V_{r}}{\partial t}-G_{k}\left(V_{r}-V_{k}\right)-\bar{g}_{l}\left(V_{r}-V_{l}\right)\right] /\left(V_{r}-V_{n a}\right) .
\end{aligned}
$$


the system (18) - (21) has a unique solution satisfying

$$
\max _{0 \leq x \leq 1}|e(x, t)| \leq C\left(V_{0}, V_{r}\right) e^{-\lambda t / C_{m}}
$$

where $C\left(V_{0}, V_{r}\right)$ is a positive constant depending $V_{0}$ and $V_{r}$. Furthermore, $V_{r}$ satisfies the partial differential equation (18).

Proof. We introduce the variable transform

$$
V=\hat{V}+X
$$

where the function $X(x, t)$ is to be determined. Substituting this transform into (18)-(20) gives

$$
\begin{aligned}
C_{m} \frac{\partial \hat{V}}{\partial t}+C_{m} \frac{\partial X}{\partial t}= & \frac{a}{2 R}\left(\frac{\partial^{2} \hat{V}}{\partial x^{2}}+\frac{\partial^{2} X}{\partial x^{2}}\right)-G_{n a}\left(\hat{V}+X-V_{n a}\right)-G_{k}\left(\hat{V}+X-V_{k}\right) \\
& -\bar{g}_{l}\left(\hat{V}+X-V_{l}\right)+I_{d}, \\
\frac{\partial(\hat{V}+X)}{\partial x}(0, t)= & r_{1}, \\
\frac{\partial(\hat{V}+X)}{\partial x}(1, t)= & r_{2}, \\
e(x, t)= & \hat{V}(x, t)+X(x, t)-V_{r}(x, t) .
\end{aligned}
$$

This system can be split into two systems

$$
\begin{aligned}
C_{m} \frac{\partial X}{\partial t} & =\frac{a}{2 R} \frac{\partial^{2} X}{\partial x^{2}}-G_{n a}\left(X-V_{n a}\right)-G_{k}\left(X-V_{k}\right)-\bar{g}_{l}\left(X-V_{l}\right)+I_{d} \\
\frac{\partial X}{\partial x}(0, t) & =r_{1}, \frac{\partial X}{\partial x}(1, t)=r_{2}, \\
X(x, t) & =V_{r}(x, t),
\end{aligned}
$$

and

$$
\begin{aligned}
C_{m} \frac{\partial \hat{V}}{\partial t} & =\frac{a}{2 R} \frac{\partial^{2} \hat{V}}{\partial x^{2}}-G_{n a} \hat{V}-G_{k} \hat{V}-\bar{g}_{l} \hat{V} \\
\frac{\partial \hat{V}}{\partial x}(0, t) & =0, \quad \frac{\partial \hat{V}}{\partial x}(1, t)=0, \\
e(x, t) & =\hat{V}(x, t) .
\end{aligned}
$$

The regulator equations $(27)$ - (29) can be solved explicitly. The boundary condition (22) on $V_{r}$ implies that $X$ satisfies the boundary condition (28). Plugging this $X$ into (27), we then solve the equation for $G_{n a}$ to obtain

$$
G_{n a}=\left(\frac{a}{2 R} \frac{\partial^{2} V_{r}}{\partial x^{2}}+I_{d}-C_{m} \frac{\partial V_{r}}{\partial t}-G_{k}\left(V_{r}-V_{k}\right)-\bar{g}_{l}\left(V_{r}-V_{l}\right)\right) /\left(V_{r}-V_{n a}\right)
$$


Plugging $G_{n a}$ into (30), we then obtain the coefficient of $\hat{V}$ as follows:

$$
-\left(\frac{a}{2 R} \frac{\partial^{2} V_{r}}{\partial x^{2}}+I_{d}-C_{m} \frac{\partial V_{r}}{\partial t}-G_{k}\left(V_{r}-V_{k}\right)-\bar{g}_{l}\left(V_{r}-V_{l}\right)\right) /\left(V_{r}-V_{n a}\right)-G_{k}-\bar{g}_{l} .
$$

To stabilize the equation (30), we set the above coefficient to be $-\lambda<0$ and solve the resulted equation to obtain

$$
G_{k}=\left[\left(\lambda-\bar{g}_{l}\right)\left(V_{r}-V_{n a}\right)-\frac{a}{2 R} \frac{\partial^{2} V_{r}}{\partial x^{2}}-I_{d}+C_{m} \frac{\partial V_{r}}{\partial t}+\bar{g}_{l}\left(V_{r}-V_{l}\right)\right] /\left(V_{k}-V_{n a}\right) .
$$

Under the feedforward controllers (33) and (34), the equation (30) becomes

$$
C_{m} \frac{d \hat{V}}{d t}=\frac{a}{2 R} \frac{\partial^{2} \hat{V}}{\partial x^{2}}-\lambda \hat{V}
$$

It is well known (see, e.g., $[19,21])$ that the solution of the equation decays exponentially:

$$
\|\hat{V}(t)\|_{H^{1}} \leq C[\hat{V}(0)] e^{-\lambda t / C_{m}}
$$

It then follows from the Sobolev inequality (see, e.g., [8, page 270]) that

$$
\begin{aligned}
\max _{0 \leq x \leq 1}|e(x, t)| & =\max _{0 \leq x \leq 1}|\hat{V}(x, t)| \\
& \leq C\|\hat{V}(t)\|_{H^{1}} \\
& \leq C[\hat{V}(0)] e^{-\lambda t / C_{m}} .
\end{aligned}
$$

This implies the estimate (25). Finally, plugging $V_{r}$ into the controlled equation (18) and using the controller (24), we obtain that

$$
\begin{aligned}
& \frac{a}{2 R} \frac{\partial^{2} V_{r}}{\partial x^{2}}-G_{n a}\left(V_{r}-V_{n a}\right)-G_{k}\left(V_{r}-V_{k}\right)-\bar{g}_{l}\left(V_{r}-V_{l}\right)+I_{d} \\
= & \frac{a}{2 R} \frac{\partial^{2} V_{r}}{\partial x^{2}}-\left[\frac{a}{2 R} \frac{\partial^{2} V_{r}}{\partial x^{2}}+I_{d}-C_{m} \frac{\partial V_{r}}{\partial t}-G_{k}\left(V_{r}-V_{k}\right)-\bar{g}_{l}\left(V_{r}-V_{l}\right)\right]-G_{k}\left(V-V_{k}\right)-\bar{g}_{l}\left(V-V_{l}\right)+I_{d} \\
= & C_{m} \frac{\partial V_{r}}{\partial t}
\end{aligned}
$$

So $V_{r}$ satisfies the partial differential equation (18). This completes the proof.

We then consider the Dirichlet boundary condition

$$
V(0, t)=V_{1}, \quad V(1, t)=V_{2}
$$

where $V_{1}$ and $V_{2}$ are constants. It is easy to see that the proof of Theorem 2.1 can be directly applied to the Dirichlet boundary condition with the boundary condition (19) being replaced by the boundary condition (35). Thus we have the following theorem. 
Table 1: Parameters of the model (18)-(21) from [14]

\begin{tabular}{l|l}
\hline$C_{m}=1 \mu \mathrm{F} / \mathrm{cm}^{2}$ & $V_{l}=10.613 \mathrm{mV}$ \\
$\bar{g}_{l}=0.3 \mathrm{mS} / \mathrm{cm}^{2}$ & $V_{n a}=115 \mathrm{mV}$ \\
$R=35.4 \Omega$ & $V_{k}=-12 \mathrm{mV}$ \\
$a=0.0238 \mathrm{~cm}$ & $V(0)=15 \mathrm{mV}$ \\
\hline
\end{tabular}

Theorem 2.2. Assume that $\lambda>0$ and the initial condition $V_{0} \in H^{1}(0,1)$. Suppose that

1. The disturbance function $I_{d}(x, t)$ is continuous.

2. The potential reference function $V_{r}(x, t)$ is continuously differentiable in $t$ and twice continuously differentiable in $x$ and satisfies the boundary condition

$$
V_{r}(0, t)=V_{1}, \quad V_{r}(1, t)=V_{2}
$$

Then, under the feedforward controllers (23) and (24), the system (18) - (21) with the boundary condition (19) being replaced by the boundary condition (35) has a unique solution satisfying

$$
\max _{0 \leq x \leq 1}|e(x, t)| \leq C\left(V_{0}, V_{r}\right) \exp \left[-\left(\frac{a k}{2 R C_{m}}+\frac{\lambda}{C_{m}}\right) t\right]
$$

where $k$ is a positive constant and $C\left(V_{0}, V_{r}\right)$ is a positive constant depending $V_{0}$ and $V_{r}$. Furthermore, $V_{r}$ satisfies the partial differential equation (18).

We may give a speculated biological interpretation about the conductance feedforward controllers (23) and (24). It might be a biologically intrinsic membrane action potential reference pattern as shown in Figure 1, which is essential for maintaining the normal functioning of the giant nerve fibre, that opens and closes the sodium and potassium channels.

\section{A numerical example}

Since the membrane current disturbance could be periodic and uniform, we take

$$
I_{d}=6+2.8 \cos (\pi t)+0.7 \sin (\pi t)-0.5 \cos (2 \pi t)-0.1 \sin (2 \pi t)
$$

The membrane action potential reference $V_{r}$ is obtained from the experimental data in the Figure 13 in the paper [14]. Fitting a Fourier polynomial into the data, we obtain

$$
\begin{aligned}
V_{r}(x, t)= & \frac{\left(r_{2}-r_{1} e^{-2}\right) e^{2 x}+\left(r_{2}-r_{1} e^{2}\right) e^{-2 x}}{2\left(e^{2}-e^{-2}\right)}+24.86+3.42 \cos (t)+49.52 \sin (t) \\
& -16.44 \cos (2 t)+6.89 \sin (2 t)-9.26 \cos (3 t)+0.83 \sin (3 t)-2.55 \cos (4 t)-5.49 \sin (4 t) .(39
\end{aligned}
$$



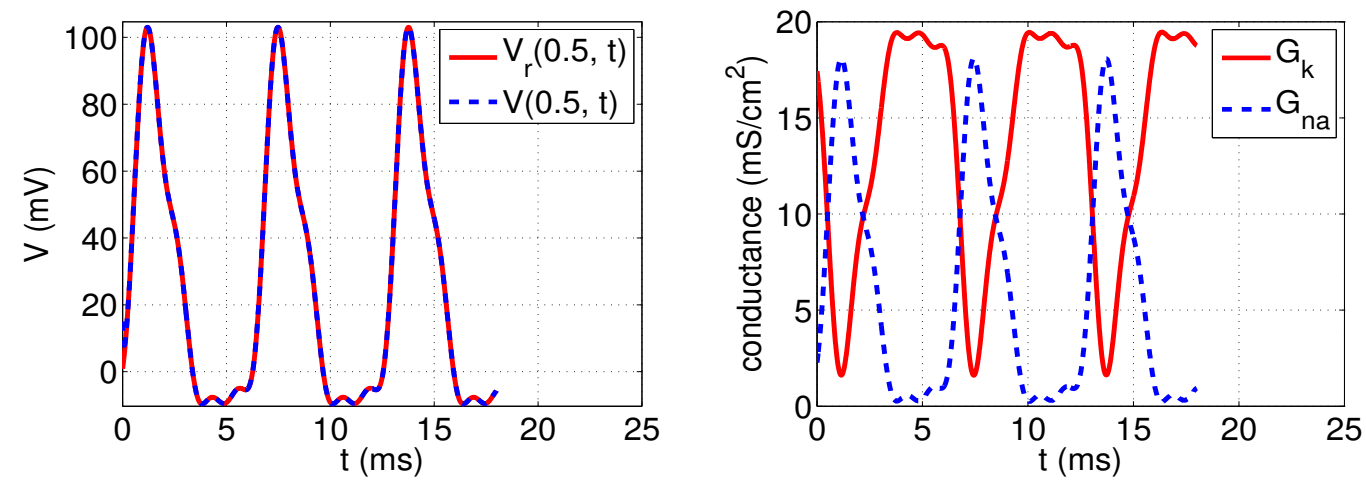

Figure 1: Left: The simulated potential $V(0.5, t)$ quickly tracks the potential reference $V_{r}(0.5, t)$ at $x=0.5$. Right: The simulated potassium and sodium conductances.

Here we have added the function of $x$ to make $V_{r}$ satisfy the boundary condition (22).

In the numerical computations, we take $\lambda=20 \mathrm{mS} / \mathrm{cm}^{2}, r_{1}=1$, and $r_{2}=5$. All other parameter values are listed in Table 1 . Then the system (18) - (21) is solved numerically by the difference method.

The left figure in Figure 1 shows that the simulated potential $V(0.5, t)$ quickly tracks the potential reference $V_{r}(0.5, t)$ at $x=0.5$. In fact, this holds for every $x \in[0,1]$. Moreover, the right figure in Figure 1 shows that the sodium conductance is close to the experimental observation in Fig. 6 in the Hodgkin and Huxley's paper [14]. When the plasma membrane is depolarized, the voltage-gated sodium channel opens rapidly and then, after about $1 \mathrm{~ms}$, inactivates. After the channel has gone through this cycle, it must spend at least $1 \mathrm{~ms}$ with the transmembrane voltage at the resting voltage before it can be opened by a second depolarization. Also the figure shows that the potassium conductance is close to the experimental observation in Figs. 2 and 3 in the Hodgkin and Huxley's paper [14]. Potassium is much more concentrated in the cytosol than outside, typically, $140 \mathrm{mmol} / \mathrm{L}$ in the cytosol but only $5 \mathrm{mmol} / \mathrm{L}$ in the extracellular medium. Thus there is a tendency for potassium ions to leave the cell down the concentration gradient. The potassium channels are the major pathway by which ions can cross the plasma membrane of an unstimulated cell. Thus, when the sodium channel opens to let sodium ions move in, the potassium ions tend to leave, and when the sodium channel shuts after about $1 \mathrm{~ms}$ and the transmembrane voltage is at the resting voltage, the potassium ions move in.

\section{References}

[1] R. Adams, Sobolev Spaces, Academic Press, New York, 1975. 
[2] K. Aihara and G. Matsumoto, Two stable steady states in the Hodgkin-Huxley Axons, Biophys. J., vol. 41, (1983), $87-89$.

[3] Brandon Ashley and Weijiu Liu, Asymptotic tracking and disturbance rejection of blood glucose regulation system, Mathematical Biosciences, 289, 2017, 78-88.

[4] E. Aulisa and D. Gilliam, A Practical Guide to Geometric Regulation for Distributed Parameter Systems, Chapman and Hall/CRC, Boca Raton, FL, 2015.

[5] L. J. Colwell and M. P. Brenner, Action potential initiation in the Hodgkin-Huxley Model, PLoS Comput Biol 5(1), (2009), e1000265.

[6] J. Deutscher, A backstepping approach to the output regulation of boundary controlled parabolic PDEs, Automatica, 57 (2015), 56-64.

[7] J. Deutscher, Backstepping design of robust output feedback regulators for boundary controlled parabolic PDEs, IEEE Trans. Autom. Control, 61 (2016), 2288-2294.

[8] L. Evans, Partial Differential Equations, American Mathematical Society, Providence, RI, 1998.

[9] J. Guckenheimer and J. S. Labouriau, Bifurcation of the Hodgkin and Huxley equations: A new twist. Bulletin Math. Biology 55, 937 (1993).

[10] M. Häusser, The Hodgkin-Huxley theory of the action potential, Nat Neurosci 3, 1165 (2000).

[11] A. L. Hodgkin and A. F. Huxley, Currents carried by sodium and potassium ions through the membrane of the giant axon of Loligo, J. Physiol. 116, (1952), 449-472.

[12] A. L. Hodgkin and A. F. Huxley, The components of membrane conductance in the giant axon of Loligo, J. Phy8iol. 1I6, (1952), 473-496.

[13] A. L. Hodgkin and A. F. Huxley, The dual effect of membrane potential on sodium conductance in the giant axon of Loligo, J. Phys8ol. 116, (1952), 497-506.

[14] Hodgkin, A. L., Huxley, A. F.: A quantitative description of membrane current and its application to conduction and excitation in nerve. J. Physicol. 117, 500-544 (1952)

[15] A. L. Hodgkin, A. F. Huxley, and B. Katz, Ionic currents underlying activity in the giant axon of the squid, Arch. Sci. physiol. 3, (1949), 129-150. 
[16] A. L. Hodgkin, A. F. Huxley, and B. Katz, Measurement of current-voltage relations in the membrane of the giant axon of Loligo, J. Physiol. 116, (1952). 424-448.

[17] A. L. Hodgkin and B. Katz, The effect of temperature on the electrical activity of the giant axon of the squid, J. Physiol. 109, (1949), 240-249.

[18] J. Huang, Nonlinear Output Regulation, Theory and Applications. Society for Industrial and Applied Mathematics, Philadelphia, 2004.

[19] I. Lasiecka, Unified theory for abstract parabolic boundary problems - a semigroup approach, Appl Math Optim 6, 287-333 (1980).

[20] H. M. Lieberstein, On the Hodgkin-Huxley partial differential equation, Mathematical Biosciences, Vol. 1, Issue 1, (1967), 45 - 69.

[21] W. Liu, Elementary Feedback Stabilization of the Linear Reaction Diffusion Equation and the Wave Equation, Mathematiques et Applications, Vol. 66, Springer, 2010.

[22] W. Liu, Introduction to Modeling Biological Cellular Control Systems. Modeling, Simulation and Applications, Vol. 6, Springer, 2012.

[23] W. Liu, Boundary feedforward and feedback control for the exponential tracking of the unstable highdimensional wave equation, Journal of Mathematical Analysis and Applications, vol 499, issue 1, July, 2021, https://doi.org/10.1016/j.jmaa.2021.125010

[24] W. Liu, Independence of convergence rate of the wave tracking error on structures of feedforward controllers, Automatica, https://doi.org/10.1016/j.automatica.2020.109264

[25] W. Liu, Feedforward boundary control for the regulation of a passive and diffusive scalar in 2-D unsteady flows, IEEE Transactions on Automatic Control, vol. 65, no. 11, pp. 4882 - 4886, 2020.

[26] W. Liu, A mathematical model for the robust blood glucose tracking. Mathematical Biosciences and Engineering, 16 (2), 2019, 759 - 781.

[27] W. Liu, Feedback and Feedforward Control of Linear Differential Equations, Unpublished lecture notes, 2021. 
[28] Florian Malchow and Oliver Sawodny, Feedforward Control of Inhomogeneous Linear First Order Distributed Parameter Systems. 2011 American Control Conference, San Francisco, CA, USA, 2011, 3597 - 3602.

[29] T. Meurer and M. Zeitz, Feedforward and Feedback Tracking Control of Nonlinear DiffusionConvection-Reaction Systems Using Summability Methods. Ind. Eng. Chem. Res. 44, 2532 - 2548 , 2005.

[30] Thomas Meurer and Andreas Kugi, Trajectory Planning and Feedforward Control Design for the Temperature Distribution in a Cuboid. Proc. Appl. Math. Mech. 6, 825 - 826, (2006)

[31] Thomas Meurer, Control of Higher-Dimensional PDEs: Flatness and Backstepping Designs. Springer, New York, 2013

[32] Thomas Meurer, Flatness-based motion planning and tracking. Lecture Notes for the Workshop "New Trends in Control of Distributed Parameter Systems" at the 2016 IEEE CDC, Las Vegas (NV), USA.

[33] V. Natarajan, D. S. Gilliam, and G. Weiss, The State Feedback Regulator Problem for Regular Linear Systems, IEEE Trans. Autom. Control 59, pp. 2708-2722 (2014).

[34] Tilman Utz and Andreas Kugi, Flatness-based feedforward control design of a system of parabolic PDEs based on finite difference semi-discretization. Proc. Appl. Math. Mech. 12, 731 - 732 (2012).

[35] Y. Zhang, K. Wang, Y. Yuan, D. Sui, H. Zhang and H. Zhang, Stability and bifurcation analysis of Hodgkin-Huxley model, 2013 IEEE International Conference on Bioinformatics and Biomedicine, Shanghai, China, 2013, pp. 49-54. 